\title{
Design and architecture of new 11:2 decimal compressors
}

\author{
PRABIR SAHA* and PUJA SAMANTA \\ National Institute of Technology Meghalaya, Shillong, India \\ e-mail: sahaprabir1@gmail.com; pooja.tuku@gmail.com
}

MS received 5 January 2018; revised 11 March 2019; accepted 19 March 2019; published online 25 April 2019

\begin{abstract}
Design and architectures of new 11:2 decimal compressors have been reported in this paper. Two design methodologies viz. delay and area optimized compressors have been introduced and implemented through tree structure of decimal number system. The architectures have been realized through vertical carry save addition algorithm, wherein to build up such addition of unconventional (4221 and 5211) binary coded decimal technique has been incorporated. Configurations of such compressors have been prototyped and transistor level implementation have been carried out to evaluate performance parameters like speed (propagation delay), power dissipation, area and area delay product. The architecture has been validated by Cadence virtuoso platform using $90 \mathrm{~nm}, 65 \mathrm{~nm}$ and $45 \mathrm{~nm}$ CMOS technology which provides useful statistics to determine the compressor's performance parameters. The propagation delay of the delay optimized compressor equals to $\sim 0.094 \mathrm{~ns}$ while the propagation delay of the area optimized compressor is $\sim 0.124 \mathrm{~ns}$ using $90 \mathrm{~nm}$ CMOS technology at $1 \mathrm{~V}$ supply voltage. The reported architectures are $\sim 24 \%$ and $\sim 41 \%$ faster from its counterpart.
\end{abstract}

Keywords. Cadence; decimal compressor; layout area; power consumption; propagation delay.

\section{Introduction}

Currently, general purpose computers are performing decimal computations through binary arithmetic [1]. However, decimal computer arithmetic is gaining interest for decimal data processing like scientific, commercial, financial and internet-based applications [2]. Though, many decimal numbers like $0.1,0.2$, etc. cannot be converted in exact binary format due to finite word-length effect, thereby errorfree computation is impracticable [3]. Recently, decimal arithmetic is becoming commercialized for general purpose computer [4], where, Binary Coded Decimal (BCD) encoding techniques maintaining the relationship between binary and decimal number system. Decimal codes are employed in such a way that the sum of the weights of each 4-bit decimal, i.e., $\sum_{i=0}^{3} r_{i}=9[5,6]$. The family of such type of codes includes $4221,5211,3321$, etc., where all the combinations are suitable for decimal arithmetic operations [5-7].

Efficient hardware implementation of multi-operand decimal addition [8-10] plays a vital role during the partial product reduction during decimal multiplication. Decimal numbers in binary format can be coded directly in digit by digit format, whereas, the addition of such BCD numbers summation exceed the decimal value 9 which creates the overflow [10]. To overcome the problem, higher order decimal compressors are helpful in reducing number of steps for partial product reduction of decimal parallel

*For correspondence multiplication; hence it reduces the overall delay of the multiplication. Moreover, almost all the parallel decimal multipliers suffer from high area requirement [11, 12], thereby the reported area optimized compressors are helpful to reduce the overall area.

In this paper, two different architecture viz. delay and area optimized 11:2 decimal compressor has been introduced using unconventional (4221 and 5211) coding techniques. This paper is an extension of a previous work of the same authors [13] and others where concept of vertical bit array compressor has been introduced which has become the building block of higher order decimal compressors. This vertical bit array compressor has made the higher order digit compressor more simple and concise. Implementation of the delay and area optimized versions of the compressors have been carried out through vertical carry save addition algorithm. The functionality of the reported architectures were checked and transistor level implementations have been carried out for practical VLSI applications. Performance parameters like propagation delay, power consumption, area and area delay product have been computed in Cadence virtuoso platform using $90 \mathrm{~nm}$ CMOS technology which provides useful statistics to evaluate the compressor's performance parameters. The results offered the pros and cons of the reported approaches.

In this work, two different 11:2 decimal compressor architectures (delay and area optimized) have been implemented and their functionalities have been examined. The basic compressors design idea along with the proposed 
implementation model has been described in section 2 . The circuit modules to build up the compressors have been discussed in section 3, followed by the results and discussions in section 4. Finally, the conclusions have been provided in section 5 .

\section{Decimal compressor architecture}

4-bit BCD-8421 is the most common representation of decimal values. The binary representation of 0 to 9 is valid in $\mathrm{BCD}$; hence, additional logic is required for the conversion (decimal value 10 to 15 ), moreover, BCD-8421 is not self-complementing. These limitations can be overcome by a family of weighted self complementing codes (4221, 5211) where sum of weights $\left(r_{3} r_{2} r_{1} r_{0}\right)$ of each 4-bit decimal is $\sum r_{j}=9$, where $\mathrm{j}$ varies from 0 to 3 unlike conventional BCD. The reduction by columns is done by compressor that adds a column of $\mathrm{m}$ digits of the same weight and produce $\mathrm{n}$ digits of adjacent weights [7, 14-16]. Mathematically the idea can be represented as:

$$
\sum_{i=0}^{m-1} x_{i}=\sum_{j=0}^{n-1} y_{j} 10^{j}
$$

The relation between $\mathrm{m}$ and $\mathrm{n}$ can be formulated as $10^{n}-1 \geq m$. A (m:n] compressor module consists $m$ inputs and $n$ output shown in figure 1 . In figure 1 , the numerical examples also considered by taking the highest decimal digit, i.e., 9, thereby highest number of rows which can be compressed into two digits should be 11 . To fully understand the concept of the (11:2] digit compressor the following mathematical equation can be formulated. The inputs are eleven digits variable $X=\left(x_{10}, x_{9}, \ldots, x_{1}, x_{0}\right)$ and the output $\mathrm{Y}$ which is of 2-bits can be formulated as:

$$
Y=\sum_{i=0}^{m-1} x_{i}=\sum_{j=0}^{n=1} y_{j} 10^{j}
$$

where $\forall\left(x_{i} \& y_{j} \in(0 . .9)\right.$.

\subsection{Decimal 3:2 compressor}

Taking a brief look at decimal carry save addition [5-7, 11], which have stabilized a known position in related designs and provided the basis for this work, indicates that a typical realization has been implemented by 4 conventional binary adder (3:2 binary compressor). Mathematical representation for the addition technique can be represented as:

$$
\begin{gathered}
A_{i}+B_{i}+\operatorname{Cin}_{i}=\sum_{i=0}^{3}\left(a_{i, j}+b_{i, j}+\operatorname{cin}_{i, j}\right) r_{i} \\
=\sum_{j=0}^{3} s_{i, j} r_{j}+2 \times \sum_{j=0}^{3} c_{i, j} r_{j}=S_{i}+2 \text { Cout }_{i}
\end{gathered}
$$

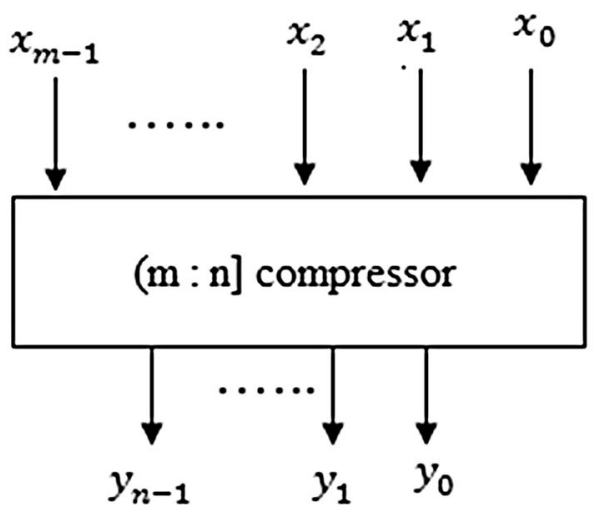

(a)

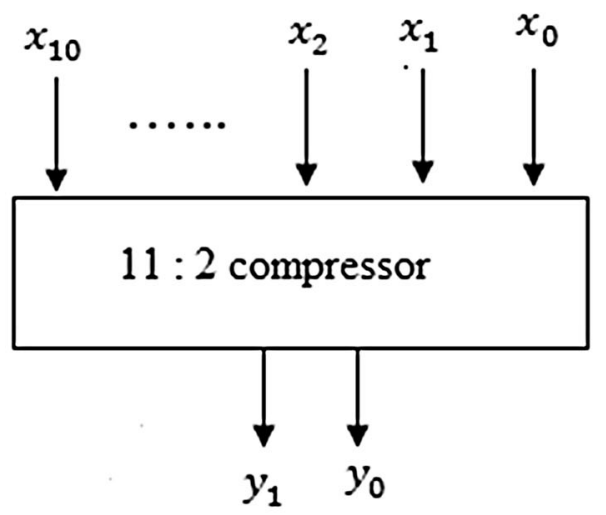

(b)

Figure 1. Compressor (a) (m:n] compressor, (b) decimal 11:2 compressor.

where $\mathrm{A}_{\mathrm{i}}, \mathrm{B}_{\mathrm{i}}$ and $\mathrm{Cin}_{\mathrm{i}}$ are defining the decimal digits, $s_{i, j}$ and $c_{i, j}$ are the sum and carry bits of full adders respectively. Cout $_{i} S_{i} \in[0,9]$, are the decimal carry and sum digits respectively, and $r$ in the digit sets based on 4211 and 5211 style. Decimal correction is not required for this 4-bit vector expressions of $\mathrm{Cout}_{i}, S_{i}$ because of coding (4221 and $5211)$ techniques. Moreover, $\times 2$ (decimal) is required for

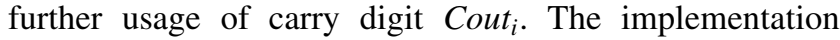
procedure (algorithmic level) for the usage of the carry bit has been shown in figure 2 [5]. In figure 2(a), a numeric example has been considered, where the implementation procedure has been described. In figure 2(b) the calculation of the carry bit has been described for the further usage of the carry bit based on the context of 5211 coding style.

The gate level implementation for the further usage of the carry bit based on 5211 coding has been described in figure 3. This architecture (figure 3) has been implemented through 4 binary full adders, combinational left shifter and digit recoder. From Eq. (3), it has been observed that decimal multiplication by 2 is required for carry digit which has been implemented through digit recoder followed by combinational left shifter [6]. Using this 3:2 digit 
Input numbers $\mathrm{A}, \mathrm{B}, \mathrm{C}$ are in

BCD 4221 format



(a)

\begin{tabular}{|c|c|c|c|c|c|}
\hline A & 5 & 1 & 0 & 0 & 1 \\
\hline$+B$ & 7 & 1 & 1 & 0 & 1 \\
\hline$+\mathrm{C}$ & 9 & 1 & 1 & 1 & 1 \\
\hline $\begin{array}{l}\text { Sum } \\
(\mathrm{S}) \\
(4221)\end{array}$ & & 1 & 0 & 1 & 1 \\
\hline \multicolumn{2}{|c|}{ Carry (4221) } & 1 & 1 & 0 & 1 \\
\hline \multicolumn{6}{|c|}{$\begin{array}{l}\text { The generated } 4221 \text { carry } \\
\text { have been re-coded into } 5221 \\
\text { format }\end{array}$} \\
\hline \multicolumn{2}{|c|}{ Carry (5221) } & 1 & 1 & 0 & 0 \\
\hline $\begin{array}{l}\text { Canry } \\
(5221) \times \\
2=\text { Canry } \\
(4211)\end{array}$ & 1 & 1 & 0 & 0 & - \\
\hline
\end{tabular}

(b)

Figure 2. (a) Flow diagram for the calculation of the carry digit for the further usage. (b) One numerical example for the carry calculation.

compressor higher order decimal compressors can be fostered. In order to build up higher order compressor, the following two rules must be adhered to:

i. All the decimal inputs of a digit compressor should follow same number system.

ii. All the decimal inputs of a digit compressor should have same digit weigtage.

\subsection{9:4 vertical bit array compressor}

A family of fast decimal digit adders has been designed by using vertical bit array compressors. Vertical bit array compressor is used to compress a larger array of bits with equal binary weights into a smaller array of bits having different binary weights. A bit array compressor sums a binary bit column of same weight and produces 3,4 or 5 weighted bits of (421), (4221) or (42221), respectively.

If we consider the same thing based on the conventional BCD coding techniques, then after every stage decimal correction stage is required. 9:4 bit array compressor has been shown in figure 4 . The architecture has also been implemented through two stages of full adders. Here $A_{i}(-$ j)are the inputs of 9:4 bit array compressor; where 'j' varies from 0 to 8 and ' $i$ ' represents the ith column of vertical bit array compressors in 9:4 digit compressor. The mathematical expressions for the implementation of the 9:4 bit array compressor are given by the following Eq. (4).

$$
\begin{aligned}
& \sum_{j=0}^{8} A_{i}(j)=2 \times\left(q_{1, i}+q_{3, i}+q_{5, i}\right)+\left(q_{0, i}+q_{2, i}+q_{4, i}\right) \\
& =Q_{i}(4221)
\end{aligned}
$$

where

$$
\begin{aligned}
& q_{0, i}=a_{i}(0) \oplus a_{i}(1) \oplus a_{i}(2) \\
& q_{1, i}=a_{i}(0) x_{i}(1)+a_{i}(1) a_{i}(2)+a_{i}(2) a_{i}(0) \\
& q_{2, i}=a_{i}(3) \oplus a_{i}(4) \oplus a_{i}(5) \\
& q_{3, i}=a_{i}(3) a_{i}(4)+a_{i}(4) a_{i}(5)+a_{i}(5) a_{i}(3) \\
& q_{4, i}=a_{i}(6) \oplus a_{i}(7) \oplus a_{i}(8) \\
& q_{4, i}=a_{i}(6) a_{i}(7)+a_{i}(7) a_{i}(8)+a_{i}(8) a_{i}(6)
\end{aligned}
$$

Thus the equation can be reformulated as:

$$
\begin{aligned}
& Q_{i}(4221)= \\
& q_{i}(0)=q_{0, i} \oplus q_{2, i} \oplus q_{4, i} \\
& q_{i}(1)=q_{0, i} q_{2, i}+q_{2, i} q_{4, i}+q_{4, i} q_{0, i} \\
& q_{i}(2)=q_{1, i} \oplus q_{3, i} \oplus q_{5, i} \\
& q_{i}(3)=q_{1, i} q_{3, i}+q_{3, i} q_{5, i}+q_{5, i} q_{1, i}
\end{aligned}
$$

It has been observed that nine binary bits of same weight are arranged in a vertical stack and compressed into four bits $\left(\mathrm{q}_{\mathrm{i}}(3), \mathrm{q}_{\mathrm{i}}(2), \mathrm{q}_{\mathrm{i}}(1), \mathrm{q}_{\mathrm{i}}(0)\right)$ with respective binary weights as $\times 4, \times 2, \times 2$ and $\times 1$. For a binary full adder the weight of the carry bit is $\times 2$, where the same for sum bit is $\times 1$. For nine bits, three binary full adders are used at the first stage; 


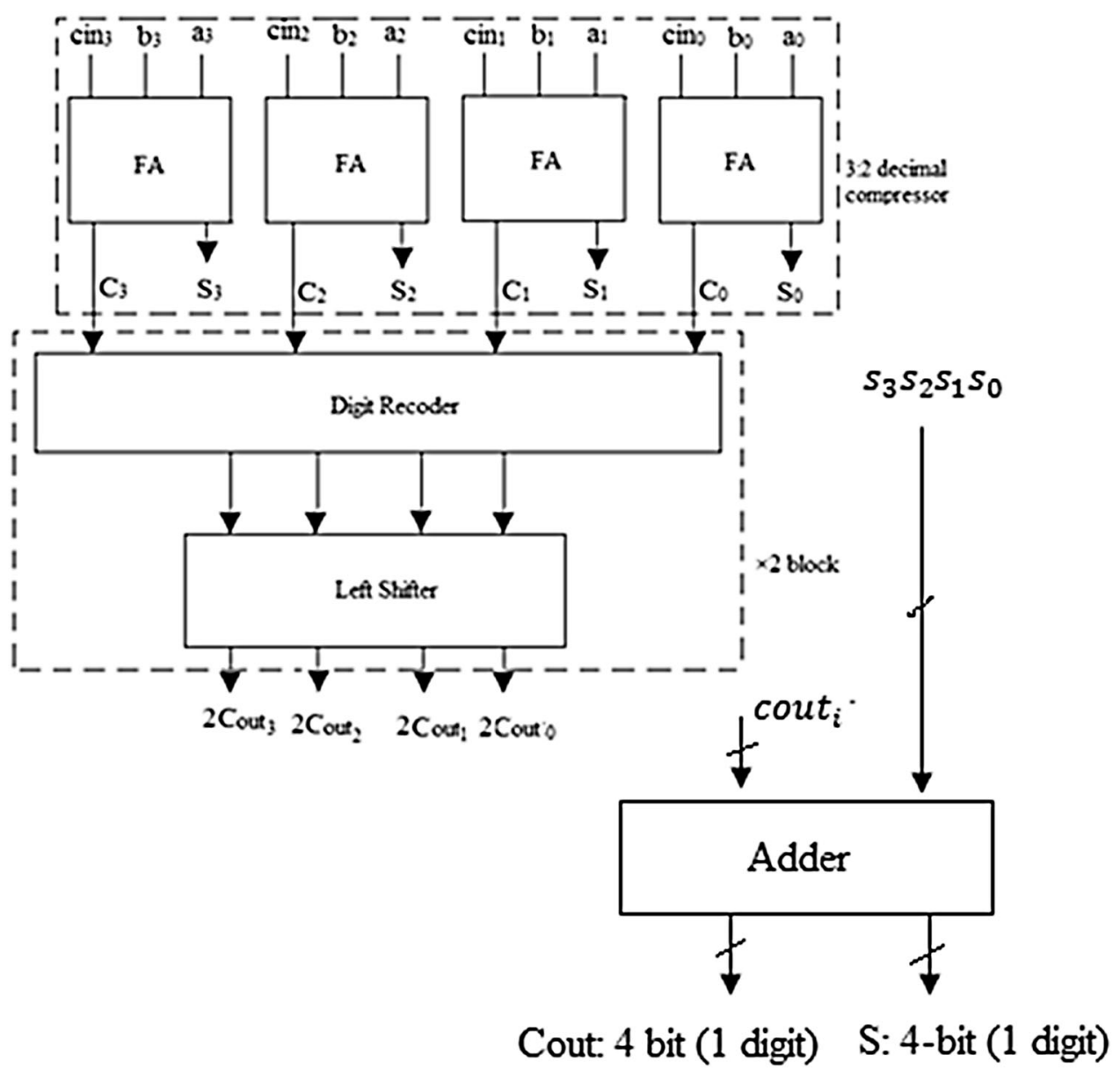

Figure 3. Architecture of basic digit compressor.

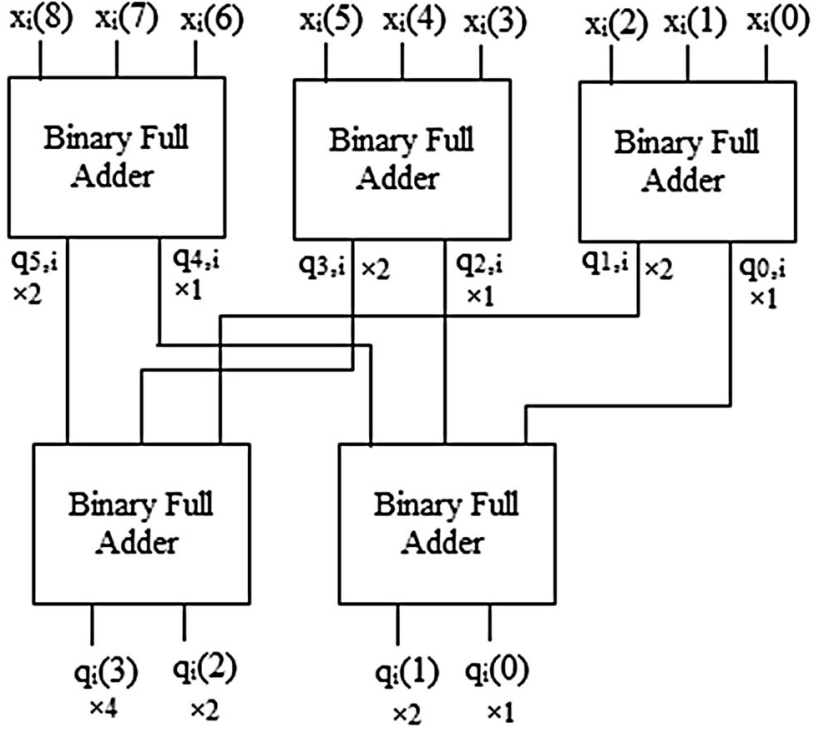

Figure 4. Architecture of 9:4 vertical bit array compressor. each binary full adder creates one carry bit $(\times 2)$ and one sum bit $(\times 1)$. So after first stage there are three bits which are having binary weightage $\times 2$ and same for weight bits $\times 1$. Thereby, three binary bits of binary weight $\times 2$ are given to another binary full adder at the second stage which results in two bits (carry and sum) of binary weights $\times 4$ and $\times 2$, respectively. Alongside three bits of binary weight $\times 1$ are also given to another 3:2 binary compressor which gives two binary bits, carry $(\times 2)$ and sum $(\times 1)$ as result. So, at the end, as a result we get a digit $\left(Q_{i}\right)$ represented in 4221 coding as in Eq. (5).

\subsection{9:4 decimal compressor}

Dot representation of 9:4 digit counters is shown in figure 5. 9:4 decimal compressor compresses nine decimal digits into four decimal digits using vertical bit array compressor. Four 9:4 vertical bit array compressors are used to design one 9:4 decimal compressor. Now, on the other hand, if we accommodate nine decimal digits (5211) in that row of four 9:4 bit array compressors in such a way 


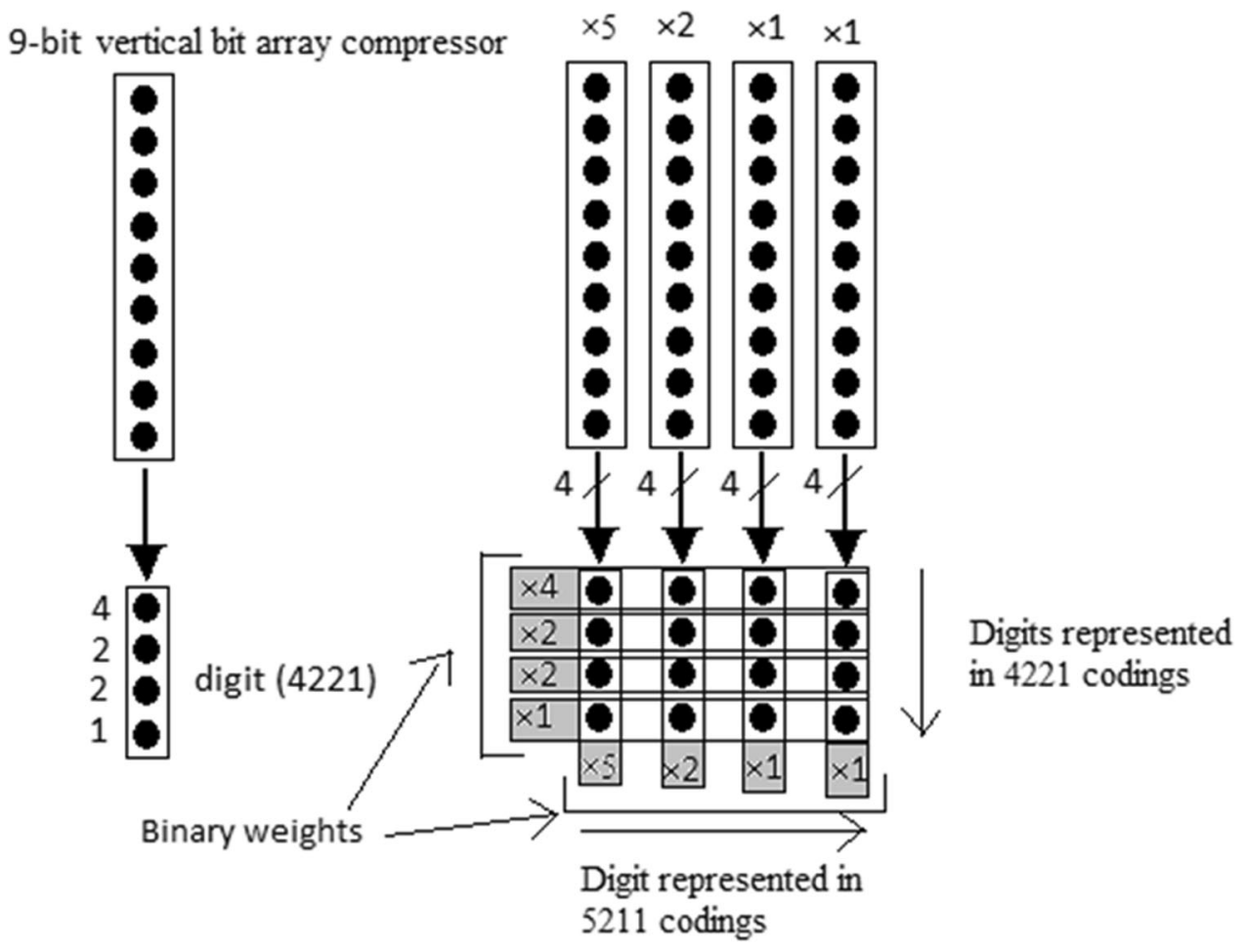

Figure 5. 9:4 decimal compressor based on 5211 coding.

that the weightage of the columns turn out to be $\times 5, \times 2$, $\times 1$ and $\times 1$ from left to right, the resultant bits will have different binary weights if we look at these from left to right and top to down. If we take resultant digits from left to right as indicated by an arrow in figure 5 , these four digits will be in 5211 coding with binary weightage $\times 4, \times 2, \times 2$ and $\times 1$.

\subsection{Proposed 11:2 delay optimized compressor}

Delay optimized 11:2 digit compressor has been shown in figure 6 . The design has been implemented through 9:4 digit compressor, basic 3:2 compressors, digit recoders and left shifters. Input digits $\left(i n_{i}\right.$; where $i$ varies from 1 to 10 and each digit is of 4 bits) of the 9:4 decimal compressor (explained in section 2.2) are in $5211(\times 5, \times 2, \times 1$ and $\times 1)$ format and the output digits $\left(\mathrm{Q}_{3}, \mathrm{Q}_{2}, \mathrm{Q}_{1}, \mathrm{Q}_{0}\right)$ are in 4221 format coding with different binary weights $(\times 4, \times 2, \times 2$ and $\times 1$ respectively) shown in the magnified view in figure 6. In magnified view $x_{k}=i n_{i}(k)$; where $k$ varies from 0 to 3 and for each value of $k, i$ varies from 1 to 10 . Another input of the compressor is $c_{i n}$. Three inputs $\left(c_{i n}, i n_{1}\right.$ and $\left.i n_{2}\right)$ in 5211 format are given as inputs of one 3:2 decimal compressor whose two outputs are also in 5211 number system and has digit weight of $\times 2$ and $\times 1$. Following the compression rules dictated in section 2.2, all the outputs with same number system and same digit weight are put together to other digit compressors for further compression.
If outputs available are not of same number system or not of same digit weight then those outputs are to be converted into some other number system or digit weight in order to compress them in the next stage and this process goes on until we end up with two digits after compression. Like $\times 5$ operation for 4221 coding can be done by left shifting of the bits of 3 times and the resulting digit with weight $\times 1$ would be in 5211 coding as sown in figure $6 . \times 2$ operation can be done by using the $\times 2$ multiplier block for 4221 coding (section 3.2a). Total five 3:2 decimal compressors have been used to design this delay optimized 11:2 decimal compressor. The weights of the sum and carry digit of digit compressors have been decided according to the weight of the input digit.

\subsection{Proposed 11:2 area optimized compressor}

Area optimized 11:2 digit compressor has been shown in figure 7. To implement this compressor only 3:2 decimal compressor (basic compressor), $\times 5$ multiplier block, $\times 2$ multiplier block, 5211-4221 recoder and 4221-5211 recoder have been used. In this architecture (figure 7) the input digits have been taken in BCD 5211 coding format. These inputs are given to a row of four 3:2 decimal compressor, as an example $C_{i n}, I_{0}$ and $\operatorname{In}_{1}$ are the input of the first (taken from right) compressor similarly $\mathrm{In}_{2}, \mathrm{In}_{3}$ and $\mathrm{In}_{4}$ are the input of the second compressor. In figure 7, basic compressors have been used to implement the higher order 


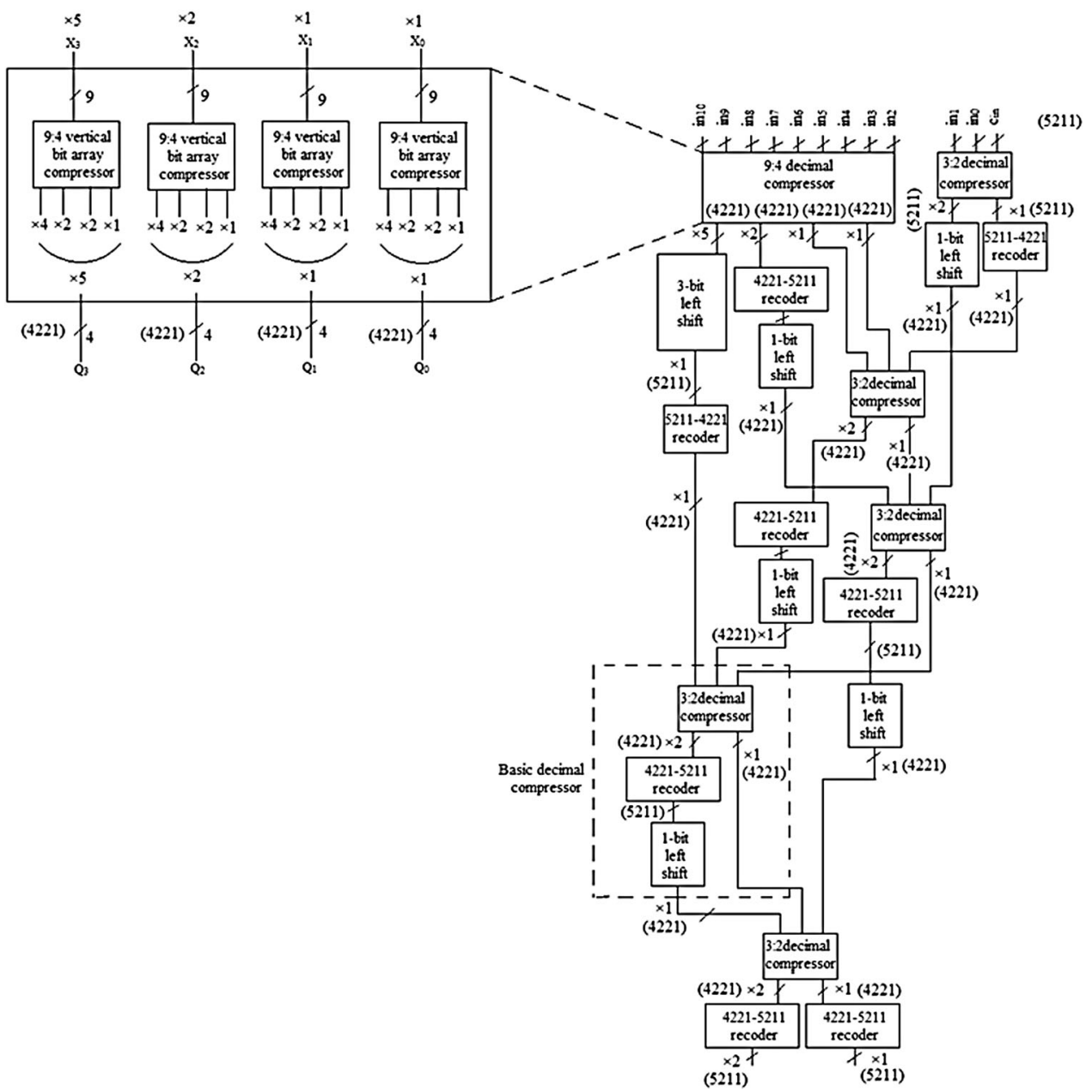

Figure 6. Proposed 11:2 delay optimized compressor.

compressors in parallel manner. $\times 5$ multiplication and $\times 2$ multiplication are the intermediate stage of the BCD 5211 and 4221 coding style. These inputs are divided in the group of three and given to a row of four 3:2 decimal compressors. Each of these compressors produces two digits; sum and carry of weights $\times 1$ and $\times 2$ respectively (as explained in section 2.1). Carry digits from the 1st stage (digit weight $\times 2$ ) are taken together and given as inputs to a 3:2 decimal compressor in 2 nd stage. The carry output of this decimal compressor has digit weight of $\times 2(\times 2)$ i.e., $\times 4$ and at the same time digit weight for the sum digit is $\times 1(\times 2)$ i.e., $\times 2$. Similarly, three digits with digit weight $\times 1$ are given to another 3:2 decimal compressor of 2 nd stage which results one sum and carry digit with weight-age of $\times 1$ and $\times 2$, respectively.

It has been observed that at the end of two 3:2 decimal compressors of second stage we get four digits (5211) with binary weight $\times 4, \times 2, \times 2$ and $\times 1$, respectively and each weighted digit consists of 4-bits, represented vertically in dot format as in figure 7. If we align the bits as sown in figure 7 and take 4-bits horizontally as a digit as represented with rectangle in the figure, then we get 4-digits which follow number system 4221 and have digit weights $\times 5, \times 2, \times 1$ and $\times 1$. Now all the signals will follow the algorithm for compression (as discussed in section 2.1) for rest of the tree; same as delay optimized compressor (figure 6). At the end, two output digits are converted into 5211 style representation to get the outputs in 5211 coding same as inputs.

\section{Circuit modules}

In this section, different circuit modules like digit recoder $\times 2$ multiplier and $\times 5$ multiplier have been described to build up the delay and area optimized 11:2 decimal compressors.

From table 1, it has been observed that number representation based on BCD 5211, and 4221 is not unique, thereby different digital circuits implementations are possible. To make it uniform digit recoder is required. Changing the intermediate results from one coding style to 


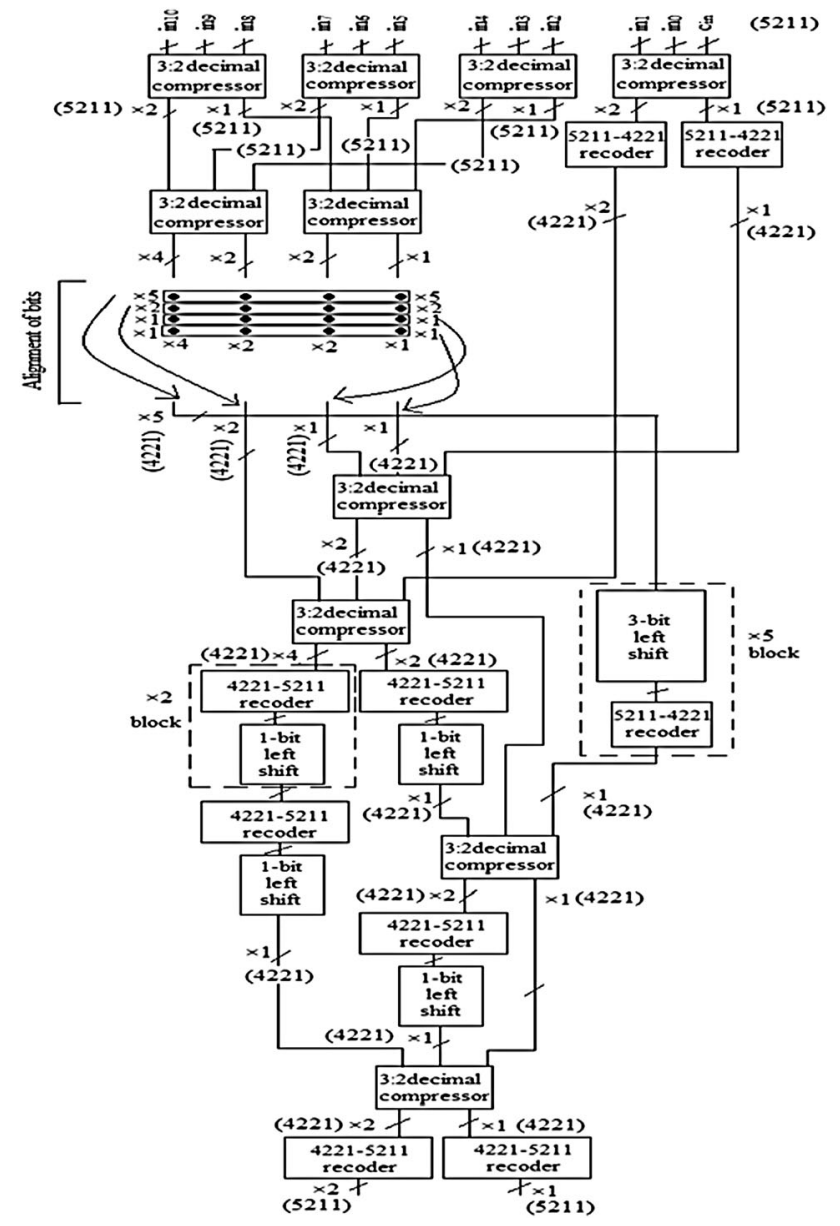

Figure 7. Area optimized decimal compressor.

Table 1. Decimal digit represented in 4221 and 5211.

\begin{tabular}{lrrrrrr}
\hline Decimal & \multicolumn{3}{c}{4221} & \multicolumn{3}{c}{5211} \\
\hline 0 & & 0000 & & & 0000 & \\
1 & 0001 & & 0001 & & 0010 \\
2 & 0100 & & 0010 & 0011 & & 0100 \\
3 & 0011 & & 0101 & 0101 & & 0110 \\
4 & 0110 & & 1000 & & 0111 & \\
5 & 0111 & & 1001 & & 1000 & \\
6 & 1010 & & 1100 & 1010 & & 1001 \\
7 & 1101 & & 1011 & 1100 & & 1011 \\
8 & & 1110 & & 1110 & & 1101 \\
9 & & 1111 & & & 1111 & \\
\hline
\end{tabular}

another like 5211 to 4221 or vice versa this digit recoder is required. Furthermore, $\times 2$ multiplier and $\times 5$ multiplier are the intermediate stage of the basic compressor as well as the higher order (11:2) compressor. The individual modules and their corresponding description including gate level implementation of the above mentioned modules have been described in this section.

\subsection{Digit recoder}

Table 1 represents the coding style of the decimal numbers based on 4221 and 5221 coding style. For each of the coding style of some numbers there are multiple representations are possible. The improved recorder is compatible for all the representations of the decimal digits based on 4221 coding.

3.1a 4221 to 5211 digit recoder: Hardware implementation of digit recoder (4221 to 5211) have been re-designed for the proposed decimal compressors, and shown in figure 8 . From table 1, it has been observed that shaded digits of (4221) coding schemes are different from (5211) coding schemes and the rest of the digit combinations are same for both cases. Thereby, shaded combinations have been treated separately and modification has been carried out for the conversion. The modification has been carried out for the following cases:

- S1: This case appears for decimal digits 4 and 5.

When the combinations appear ' 0110 ' and '0111' then value of $\mathrm{S} 1$ has been considered as high else low.

- S2: This case appears for decimal digits 4 and 5.

When the combinations appear '1000' and '1001' then value of $\mathrm{S} 2$ has been considered as high or low.

- S3: This case appears for decimal digits 6 and 7.

Either of the shaded combination appears then S3 become high or low.

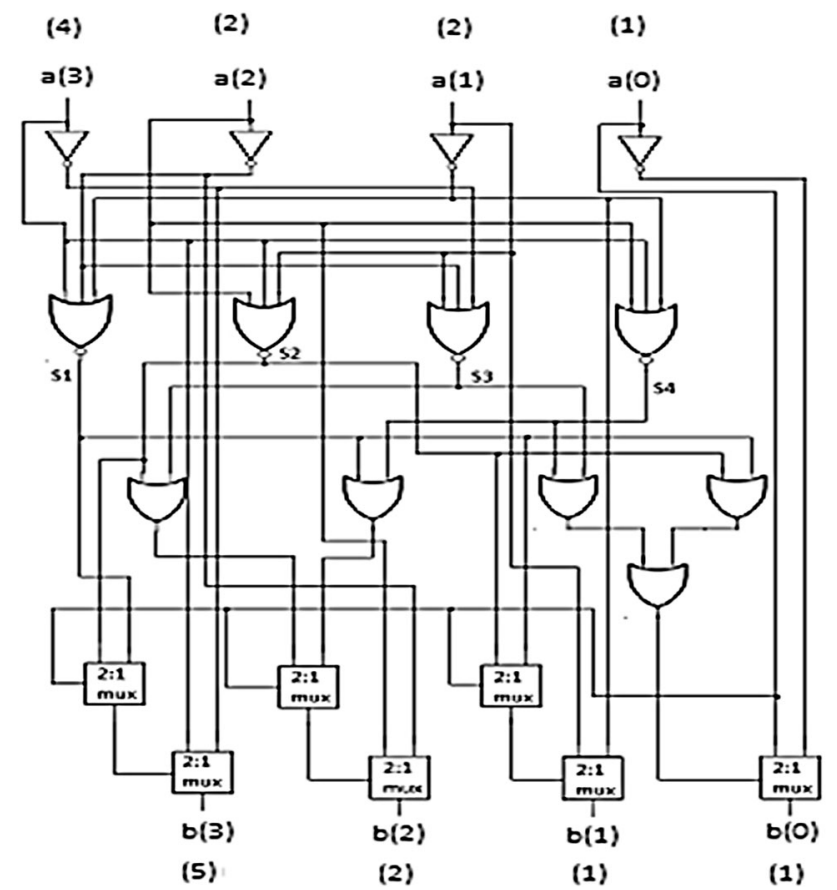

Figure 8. 4221 to 5211 recoder. 
- S4: This case appears for decimal digits 2 and 3.

Either of the shaded combination appears then S4 become high else low.

For non-shaded combinations (table 1) no modification has been carried out because they are same for both the coding techniques. After identification of the digit combinations, the value has been promoted towards multiplexers, for bit modification facilitation 4221 to 5211 conversions. This proposed digit recoder gives us flexibility to use all the sixteen combinations of each coding techniques unlike $[5,6]$ where number of combinations of decimal digit representation considered, was restricted. The same digit recoder can be used when the input digits of decimal compressors are in 4221 code and also when inputs are coded in 5211. The expressions of converting $4221\left(a_{3} a_{2} a_{1}\right.$ $\left.a_{0}\right)$ to $5211\left(b_{3} b_{2} b_{1} b_{0}\right)$ is given in Eq. (6).

$$
\begin{aligned}
& b(0)=[\overline{a(3) a(2)}+a(2)(a(1) \oplus a(3))] \oplus a(0) \\
& b(1)=[\overline{a(3)}(\overline{a(0) a(1) a(2)}+a(0) a(1) a(2))] \oplus a(1) \\
& b(2)=[\overline{a(0)}(\overline{a(1)}(a(2) \Theta a(3)))+a(0) \overline{(a(3)} a(1))] \oplus a(2) \\
& b(3)=[\overline{a(3)}(\overline{a(0) a(1) a(2)}+a(0) a(1) a(2))] \oplus a(3)
\end{aligned}
$$

3.1b 5211 to 4221 digit recoder: Figure 9 shows a digit recoder of 5211 to 4221 coding. The output weighted bit $\times 4$ is same as input weighted bit $\times 5$ and one of the weighted bit $\times 2$ of the output digit is same as the $\times 2$ weighted bit of the input digit. Another $\times 2$ weighted bit of the output digit comes from the output of one 2:1 MUX as shown in figure 10 . This bit is basically the carry output of the full adder whose corresponding inputs are $\times 5, \times 1$ and $\times 1$ weighted bits of the input digit (5211) of the digit recoder. The output $\times 1$ bit is the sum of the above mentioned full adder as shown in figure 9. The mathematical expression of 5221 to 4211 recoding scheme has been shown in Eq. (7).



Figure 9. 5211 to 4221 digit recoder.

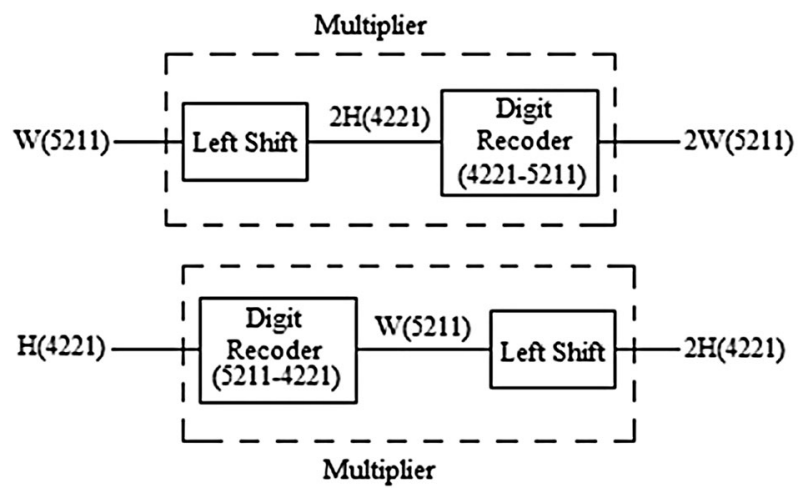

Figure 10. $\times 2$ multiplication block.

$$
\begin{aligned}
& b(0)=a(0) \oplus a(1) \oplus a(3) \\
& b(1)=a(1) \bullet \overline{(a(0) \oplus a(1))}+a(3) \bullet(a(0) \oplus a(1)) \\
& b(2)=a(2) \\
& b(3)=a(3)
\end{aligned}
$$

\subsection{Multiplier}

3.2a $\times 2$ multiplication: From Eq. (3), it has been observed that one decimal $\times 2$ operation is needed for decimal basic compressor. Multiplication of $\times 2$ block diagram is shown in figure 10. If the input to the multiplier block is in 4221 coding then the multiplier needs one 4221 to 5211 digit recoder first and then one bit left shift block. In case of 5211 coding, one left shift block is required first and then one 4221-5211 digit recoder to perform $\times 2$ decimal multiplication.

$3.2 \mathrm{~b} \times 5$ multiplication: Figure 11 (a) shows an example where $\mathrm{H}$ has been represented through 4221 coding scheme and the multiplication of the same digit by 5 has been represented by $\mathrm{W}$ (5211 coding scheme). If $\mathrm{H}$ is left shifted by 3 bits, we can get 5 times of H (5211 coding) i.e., $5 \mathrm{~W}$. Thereby, one $\times 5$ multiplication block consists of three cascaded 1-bit left shift blocks shown in figure 11(b). This multiplication block takes input in 4221 coding and gives output in 5211 coding. If the output need to be converted into 4221 coding, one 5211 to 4221 digit recoder (figure 9) is required.

\section{Comparison}

In this paper, transistor level implementation of the reported architecture has been carried out and their functionality has been checked. Implementation has been carried out for the further usage of the compressors in different circuits. One of the applications of this 




(a)

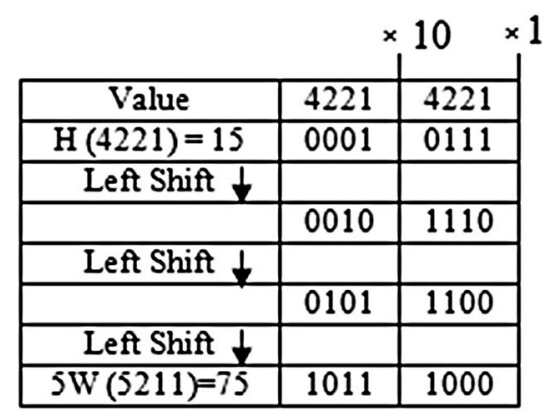

(b)

Figure 11. (a) Architecture of the $\times 5$ multiplication block. (b) A numerical example, in this example input has been considered in decimal 4221 coding and output have been observed in decimal 5211 coding.

compressor circuit is multi-operand decimal adders. The details of the multi-operand decimal adders are shown in section 2.1. The references have been taken from the different sources and have been implemented using same technological environment. Cadence Virtuoso platform has been used for the simulation of the above mentioned compressors. The simulation results are compared with $[5,7]$ architectures and shown in tables 2 and 3. From tables 2 and 3, it has been observed that [5] has the superior results than [7], thereby, comparisons have been made with respect to [5].

\subsection{Simulation environment}

The decimal 11:2 compressors have been synthesized using Cadence Virtuoso platform with the library file of gpdk of $90 \mathrm{~nm}, 65 \mathrm{~nm}$ and $45 \mathrm{~nm}$ CMOS technology in room temperature. We have also implemented the reference architecture under the same environmental condition for the comparison. Input data was taken in a regular fashion for experimental purpose. For each transition, the delay is measured from $50 \%$ of the input voltage swing to $50 \%$ of the output voltage swing and the average value has been reported.

Table 2. Performance parameter like as propagation delay and power consumption analysis of the 11: 2 delay optimized compressor.

\begin{tabular}{|c|c|c|c|c|c|c|c|c|c|c|}
\hline \multirow[b]{2}{*}{$\begin{array}{l}\text { Supply voltage/ } \\
\text { architecture }\end{array}$} & \multicolumn{2}{|c|}{$1 \mathrm{~V}$} & \multicolumn{2}{|c|}{$1.5 \mathrm{~V}$} & \multicolumn{2}{|c|}{$2.0 \mathrm{~V}$} & \multicolumn{2}{|c|}{$2.5 \mathrm{~V}$} & \multicolumn{2}{|c|}{$3.0 \mathrm{~V}$} \\
\hline & $\begin{array}{l}\text { Delay } \\
\text { (ns) }\end{array}$ & $\begin{array}{l}\text { Power } \\
(\mathrm{mW})\end{array}$ & $\begin{array}{l}\text { Delay } \\
\text { (ns) }\end{array}$ & $\begin{array}{l}\text { Power } \\
(\mathrm{mW})\end{array}$ & $\begin{array}{l}\text { Delay } \\
\text { (ns) }\end{array}$ & $\begin{array}{l}\text { Power } \\
(\mathrm{mW})\end{array}$ & $\begin{array}{l}\text { Delay } \\
\text { (ns) }\end{array}$ & $\begin{array}{l}\text { Power } \\
(\mathrm{mW})\end{array}$ & $\begin{array}{c}\text { Delay } \\
\text { (ns) }\end{array}$ & $\begin{array}{l}\text { Power } \\
(\mathrm{mW})\end{array}$ \\
\hline \multicolumn{11}{|c|}{ (a) Delay and power analysis based on $90 \mathrm{~nm}$ CMOS technology file } \\
\hline Ref. [5] & 0.124 & 10.079 & 0.182 & 11.011 & 0.267 & 16.850 & 0.298 & 19.455 & 0.301 & 22.810 \\
\hline Ref. [7] & 0.137 & 11.023 & 0.196 & 12.053 & 0.293 & 17.082 & 0.310 & 21.054 & 0.323 & 24.055 \\
\hline Proposed & 0.094 & 10.077 & 0.176 & 11.179 & 0.260 & 16.923 & 0.282 & 20.367 & 0.285 & 23.068 \\
\hline \multicolumn{11}{|c|}{ (b) Delay and power analysis based on $65 \mathrm{~nm}$ CMOS technology file } \\
\hline Ref. [5] & 0.113 & 11.52 & 0.163 & 11.89 & 0.241 & 17.69 & 0.278 & 21.57 & 0.298 & 23.13 \\
\hline Ref. [7] & 0.117 & 11.93 & 0.183 & 12.39 & 0.253 & 18.79 & 0.299 & 23.01 & 0.321 & 25.32 \\
\hline Proposed & 0.089 & 11.50 & 0.159 & 11.87 & 0.229 & 18.09 & 0.253 & 22.36 & 0.296 & 24.67 \\
\hline \multicolumn{11}{|c|}{ (c) Delay and power analysis based on $45 \mathrm{~nm}$ CMOS technology file } \\
\hline Ref. [5] & 0.107 & 12.12 & 0.149 & 12.99 & 0.23 & 18.59 & 0.259 & 23.73 & 0.285 & 25.32 \\
\hline Ref. [7] & 0.113 & 12.93 & 0.157 & 13.38 & 0.27 & 20.52 & 0.31 & 25.36 & 0.331 & 28.97 \\
\hline Proposed & 0.085 & 12.57 & 0.148 & 13.17 & 0.22 & 19.72 & 0.268 & 24.79 & 0.283 & 26.98 \\
\hline
\end{tabular}


Table 3. Performance parameter like as propagation delay and power consumption analysis of the 11: 2 area optimized compressor.

\begin{tabular}{|c|c|c|c|c|c|c|c|c|c|c|}
\hline \multirow[b]{2}{*}{$\begin{array}{l}\text { Supply voltage/ } \\
\text { architecture }\end{array}$} & \multicolumn{2}{|c|}{$1 \mathrm{~V}$} & \multicolumn{2}{|c|}{$1.5 \mathrm{~V}$} & \multicolumn{2}{|c|}{$2.0 \mathrm{~V}$} & \multicolumn{2}{|c|}{$2.5 \mathrm{~V}$} & \multicolumn{2}{|c|}{$3.0 \mathrm{~V}$} \\
\hline & $\begin{array}{c}\text { Delay } \\
\text { (ns) }\end{array}$ & $\begin{array}{l}\text { Power } \\
(\mathrm{mW})\end{array}$ & $\begin{array}{c}\text { Delay } \\
\text { (ns) }\end{array}$ & $\begin{array}{l}\text { Power } \\
(\mathrm{mW})\end{array}$ & $\begin{array}{c}\text { Delay } \\
\text { (ns) }\end{array}$ & $\begin{array}{l}\text { Power } \\
(\mathrm{mW})\end{array}$ & $\begin{array}{c}\text { Delay } \\
\text { (ns) }\end{array}$ & $\begin{array}{l}\text { Power } \\
(\mathrm{mW})\end{array}$ & $\begin{array}{c}\text { Delay } \\
\text { (ns) }\end{array}$ & $\begin{array}{l}\text { Power } \\
(\mathrm{mW})\end{array}$ \\
\hline \multicolumn{11}{|c|}{ (a) Delay and power analysis based on $90 \mathrm{~nm}$ CMOS technology file } \\
\hline Ref. [5] & 0.219 & 10.082 & 0.277 & 11.088 & 0.345 & 17.090 & 0.372 & 20.071 & 0.413 & 23.231 \\
\hline Ref. [7] & 0.225 & 10.21 & 0.281 & 11.520 & 0.351 & 17.910 & 0.387 & 20.815 & 0.429 & 23.00 \\
\hline Proposed & 0.198 & 10.09 & 0.232 & 10.986 & 0.269 & 16.265 & 0.304 & 19.391 & 0.301 & 22.709 \\
\hline \multicolumn{11}{|c|}{ (b) Delay and power analysis based on $65 \mathrm{~nm}$ CMOS technology file } \\
\hline Ref. [5] & 0.20 & 11.12 & 0.253 & 11.97 & 0.301 & 19.01 & 0.345 & 21.71 & 0.39 & 24.23 \\
\hline Ref. [7] & 0.21 & 11.52 & 0.263 & 12.04 & 0.312 & 20.01 & 0.353 & 21.99 & 0.41 & 24.93 \\
\hline Proposed & 0.18 & 11.1 & 0.229 & 11.13 & 0.267 & 18.1 & 0.315 & 20.39 & 0.35 & 23.7 \\
\hline \multicolumn{11}{|c|}{ (c) Delay and power analysis based on $45 \mathrm{~nm}$ CMOS technology file } \\
\hline Ref. [5] & 0.19 & 11.92 & 0.23 & 12.53 & 0.283 & 19.0 & 0.345 & 22.89 & 0.361 & 25.23 \\
\hline Ref. [7] & 0.20 & 12.10 & 0.24 & 12.93 & 0.291 & 19.97 & 0.351 & 23.13 & 0.384 & 26.23 \\
\hline Proposed & 0.17 & 11.23 & 0.18 & 11.98 & 0.237 & 18.1 & 0.29 & 21.79 & 0.31 & 24.79 \\
\hline
\end{tabular}

\subsection{Critical path delay}

The critical path delay of the proposed delay optimized 11:2 compressor (figure 6) consists of one 9:4 decimal compressor, 2 numbers of 1-bit left shifter, three numbers of 3:2 digit compressors and three numbers of $\times(4221$ 5211 digit recoder). 3:2 digit compressor (figure 3) has been implemented through full adder, digit recoder and left shifter. Critical path delay of full adder approximated as $\sim 2$ XOR gate. Digit recoder (figure 8 ) has the critical path delay of $\sim 3$ XOR gate. And 1-bit left shifter has 1 XOR gate delay. Thus, overall 3:2 basic compressor has 6 XOR gate delay. It has been (figure 4) observed that vertical bit has been implemented in 2 stage of full adder, thus critical path delay is equals to 4 XOR gate. 9:4 digit compressors (figure 5) consists of 9:4 vertical bit array compressor and 4221-5211 decoder. 4221-5211 (figure 8) has a critical path delay $\sim 8$ XOR gate. Thereby critical path delay for the $9: 4$ digit compressor is $\sim 13$ XOR gate. Thereby total critical path delay of the delay optimized 11:2 compressor is equal to $(13+2+3 \times 6+3 \times 8) 57$ XOR gate delay. Similarly, critical path delay of the area optimized 11:2 compressor (figure 7) have been implemented by two 1-bit left shifter, six number 3:2 digit compressor and four number 4221-5211 digit recoder. Thereby total critical path delay $(2 \times 1+6 \times 6+4 \times 8) \sim 70$ XOR gate delay.

\section{Results}

Performance parameters as a function of propagation delay and dynamic power consumption [17] analysis of the proposed delay optimized 11:2 compressor architecture have been shown in table 2. Table $2 \mathrm{a}$ is based on $90 \mathrm{~nm}$ CMOS technology [18-22], table $2 \mathrm{~b}$ is based on $65 \mathrm{~nm}$ technology file [22] and figure $2 \mathrm{c}$ is based on $45 \mathrm{~nm}$ technology file [22]. Supply voltage has been varied linearly from $1 \mathrm{~V}$ to
$3 \mathrm{~V}$ with the linear increment of $0.5 \mathrm{~V}$ step. From table 2a it has been observed that propagation delay of the proposed compressor is $0.094 \mathrm{~ns}$, when the supply voltage is $1 \mathrm{~V}$, at $90 \mathrm{~nm}$ CMOS technology. Moreover, the power consumption of the same at $1 \mathrm{~V}$ supply is $10.077 \mathrm{mw}$. The power is comparable with the reference paper. But the compressor is $\sim 24 \%$ faster (less propagation delay) from the reference voltage. When the supply voltage is increasing then also maintains the same ratio $\sim 25 \%$ is lesser than the reference compressor. When the performance parameters are measuring at $65 \mathrm{~nm}$ technology file at $1 \mathrm{~V}$ supply, propagation delay is only $0.089 \mathrm{~ns}$ and this compressor is $\sim 22 \%$ faster than the reference compressor, and the power is almost equal. Same thing is happening when results are compared at $45 \mathrm{~nm}$ technology. The details chart of the implementation is shown in table 2 .

Table 3 is also describing the propagation delay and power consumption analysis of the area optimized compressor. Table 3a is based on $90 \mathrm{~nm}$ CMOS technology [18-22], table $3 \mathrm{~b}$ is the based on $65 \mathrm{~nm}$ technology file [22] and figure $3 \mathrm{c}$ is based on $45 \mathrm{~nm}$ technology file [22]. Dynamic switching power can be can be calculated theoretically as $P=\frac{1}{T} \int_{0}^{T} I_{d}(t) V_{D D}(t) d t$, where T is the switching time interval of the circuit [17]. In this area optimized compressor, at $1 \mathrm{~V}$ power supply, the propagation delay is $0.198 \mathrm{~ns}$, still the compressor is $\sim 9 \%$ faster than the reference architecture and the power consumption is almost equal with respect to their counterpart an $90 \mathrm{~nm}$ CMOS technology. Increasing the supply voltage the propagation delay is reducing $\sim 7 \%$ to $\sim 10 \%$. The power is comparable with the reference architecture. Using the $65 \mathrm{~nm}$ and $45 \mathrm{~nm}$ technology file, the propagation delay is reducing by $\sim 7 \%$ to $10 \%$, and the power is comparable with the reference architecture.

Table 4 describes the layout area of the proposed and the reference compressor. Area delay product measurement of the compressors has been shown in table 4 . 
Table 4. Area comparison.



\section{Discussions}

From table 2, it has been observed that dynamic switching power consumption is slightly higher for this proposed architecture as compared to the reference architecture as a function of the supply voltage. The propagation delay of the delay optimized compressor equals to $0.094 \mathrm{~ns}$ (table 2), while the propagation delay of the area optimized compressor is $0.128 \mathrm{~ns}$ (table 3 ) at $1 \mathrm{~V}$ supply voltage. The proposed compressor is $\sim 24 \%$ faster than the reference compressor in case of delay optimized (table 2) design. While, for the area optimized case the proposed methodology is $\sim 41 \%$ faster from its counterpart (table 3 ) at $1 \mathrm{~V}$ supply voltage. The propagation delay comparison among two different proposed methodology (i.e., delay and area optimized compressors) delay optimized compressor is $\sim 26 \%$ faster from the area optimized compressor. To take the view point of area delay product (table 4) it has been observed that delay optimized compressor is more beneficial with respect to delay and area measurement (table 5).
Table 5. Performance parameter analysis as a function of area delay product.

\begin{tabular}{lccccc}
\hline \multirow{2}{*}{$\begin{array}{l}\text { Supply voltage/ } \\
\text { architecture }\end{array}$} & $1 \mathrm{~V}$ & $1.5 \mathrm{~V}$ & $2.0 \mathrm{~V}$ & $2.5 \mathrm{~V}$ & $3.0 \mathrm{~V}$ \\
\cline { 2 - 6 } & & & & & \\
\hline $\begin{array}{l}\text { Delay optimized } \\
\quad \text { Ref. [5] }\end{array}$ & 0.582 & 0.855 & 1.254 & 1.400 & 1.414 \\
$\quad \begin{array}{l}\text { Proposed } \\
\text { Area optimized }\end{array}$ & 0.357 & 0.668 & 0.988 & 1.071 & 1.083 \\
$\quad$ Ref. [5] & 0.700 & 0.886 & 1.104 & 1.190 & 1.321 \\
$\quad$ Proposed & 0.516 & 0.720 & 1.108 & 1.125 & 1.152 \\
\hline
\end{tabular}

\section{Conclusions}

In this paper, design and architectures of new 11:2 decimal compressors have been reported. Transistor level implementations of such compressors have been carried out and functionalities have been verified in Cadence Virtuoso platform. Performance parameters as a function of delay, 
power and area have been calculated through Cadence with the library file of gpdk of $90 \mathrm{~nm}, 65 \mathrm{~nm}$ and $45 \mathrm{~nm}$ CMOS technology. Simulation results offered that propagation delay of the delay optimized compressor is $\sim 0.094 \mathrm{~ns}$ (at $90 \mathrm{~nm}$ CMOS technology with a supply voltage $1 \mathrm{~V}$ ) and $\sim 24 \%$ faster from its counterpart. To take the view point of area delay product, it has been observed that delay optimized compressor is more economical from its counterpart and can be a prominent solution for the decimal computing system.

\section{References}

[1] James R K, Shahana T K, Jacob K P and Sasi S 2008 Fixed point decimal multiplication using RPS algorithm. In: Proceedings of the International Symposium on Parallel and Distributed Processing with Applications, ISPA '08, pp. 343-350

[2] Cowlishaw M F 2003 Decimal floating-point: algorism for computers. In: Proceedings of the 16th IEEE Symposium on Computer Arithmetic, Santiago de Compostela, pp. 104-111

[3] Véstias M P and Neto H C 2010 Parallel decimal multipliers using binary multipliers. In: Proceedings of the Southern Programmable Logic Conference (SPL), pp. 73-78

[4] Jaberipur G and Kaivani A 2007 Binary-coded decimal digit multipliers. IET J. Comput. Digit. Tech. 1(4): 377-381

[5] Vazuez A, Antelo E and Montuschi P 2010 Improved design of high-performance parallel decimal multipliers. IEEE Trans. Comput. 59(5): 679-693

[6] Vazquez A, Antelo E and Montuschi P 2007 A new family of high performance parallel decimal multipliers. In: 18th IEEE Symposium on Computer Arithmetic, pp. 195-204

[7] Castellanos I D and Stine J E 2008 Compressor trees for decimal partial product reduction. In: Proceedings of the ACM 18th ACM Great Lakes Symposium on VLSI (GLSVLSI '08), pp. 107-110

[8] Kenney R D and Schulte M J 2004 Multioperand decimal addition. In: Proceedings of the IEEE Computer Society Annual Symposium on VLSI, pp. 251-253
[9] Kenney R D and Schulte M J 2004 High-speed multioperand decimal adders. IEEE Trans. Comput. 54(8): 953-963

[10] Dadda L and Nannarelli A 2009 Fast Multi Operand Decimal Adders Using Digit Compressors with Decimal Carry Generation. Technical University of Denmark, 2009 (IMM Technical Report-2009-05)

[11] Veeramachaneni S, Krishna M K, Avinash L, Reddy S and Srinivas M B 2007 Novel, high-speed 16-digit BCD adders conforming to IEEE 754r format. In: IEEE Computer Society Annual Symposium on VLSI (ISVLSI '07), pp. 43-50

[12] Gonzalez-Navarro S, Tsen C and Schulte M J 2013 Binary integer decimal-based floating-point multiplication. IEEE Trans. Comput. 62(7): 1460-1463

[13] Saha P, Samanta P and Kumar D 2016 4:2 and 5:2 Decimal compressors. In: Proc. 7th Int. Conf. on Intelligent Systems, Modelling and Simulation, pp. 424-429

[14] Erle M A, Hickmann B J and Schulte M J 2009 Decimal floating-point multiplication. IEEE Trans. Comput. 58(7): 902-916

[15] Richards R K 1955 Arithmetic operations in digital computers. Princeton, NJ: Van Nostrand Company

[16] Ercegovac M D and Lang T 2004 Digital Arithmetic. San Francisco: Morgan Kaufman

[17] Uyemura J P 2001 CMOS Logic Circuit Design. Kluwer, New York

[18] Zhao W and Cao Y 2006 New generation of Predictive Technology Model for sub-45 nm early design exploration. In: International Symposium on Quality Electronic Design (ISQED'06), pp. 590-596

[19] Cao Y, Sato T, Sylvester D, Orshansky M and Hu C 2000 New paradigm of predictive MOSFET and interconnect modeling for early circuit design. In: Proceedings of the Custom Integrated Circuits Conference, pp. 201-204

[20] Ueno K 2011 Material and process challenges for interconnects in nanoelectronics era. In: Int. Symposium on VLSI Technology, Systems and Applications (VLSI-TSA), pp. 64-65

[21] Kosonocky S, Burd T, Kasprak K, Schultz R and Stephany R 2012 Designing in scaled technologies: $32 \mathrm{~nm}$ and beyond. In: Symposium on VLSI Technology (VLSIT), pp. 147-148

[22] http://ptm.asu.edu/latest.html 